\title{
Patient treatment prediction by continuous time random walk inside complex system
}

\author{
Andrzej Walczak* \\ Institute of Computer and Information Systems, Military University of Technology, Warsaw, gen. Urbanowicza St.2, Poland
}

\begin{abstract}
Stochastic resonance model for medical patient condition is proposed. Approach has been generalized by means of fractional Fokker-Planck equation and subdiffusion processes. Nonadditive entropy method has been used to achieve nonlinear fractional Fokker-Planck equation. We proved that duration of an unchanged patient situation can be estimated and fulfills rules for "fat tail" probability distribution. We also proved that probability of patient staying in an unchanged condition behaves the same. Formal rules were built on concept of similarity between real patient condition and potential well model. Such approach is new and allows new results as alternative for discrete models of prediction. Obtained results get probability for patient health parameter behavior in really detailed way.
\end{abstract}

\section{Introduction}

Time series are quite obvious result of medical patient data registration case. Usually processes of registration are discrete and are not regular in time but in fact all registered data are continuous functions of time. So random walking in continuous time along queue (CTRW- Continuous Time Random Walk along queue) seems adequate model for considered data processing. Markov chains and hidden Markov chains are quite popular tools for time series analysis also in medical predictions [1]. We propose to use more general approach in form of Fokker-Planck (FP) equation. Also stochastic resonance phenomena are part of explanation the main idea of patient condition math modeling in terms of FP equation. Stochastic resonance explains rules of movement inside queue with continuous time.

As a picture we use some data obtained in research project created for cardiology [2]. Chosen data are presented in the figure 1. Measured medical parameters shown in the figure means:
- SBP and DBP are blood pressure during proper heart beat (diastolic and systolic),

- HR- heart beat per minute,

- TFC - total fluid control,

- Delta TFC- increase or decrease of TFC,

- Delta TBW - variation of Total Body Weight.

Each measured parameter is divided into limited section of values (see fig.1). Such sections are recognized in medicine as connected with patient condition level. One can assume that such section describes kind of potential well. Each well means appropriate level of patient condition. Jump from one section to another is in fact movement over barrier similarly to jump from one potential well to another (see figure 2). There are some characteristic features:

1. No one knows how long patient parameters belong to the particular well,

2. Mutual dependency of such durations in each separate well is generally unknown,

3. Detailed mechanisms of movement between such wells are not known. 


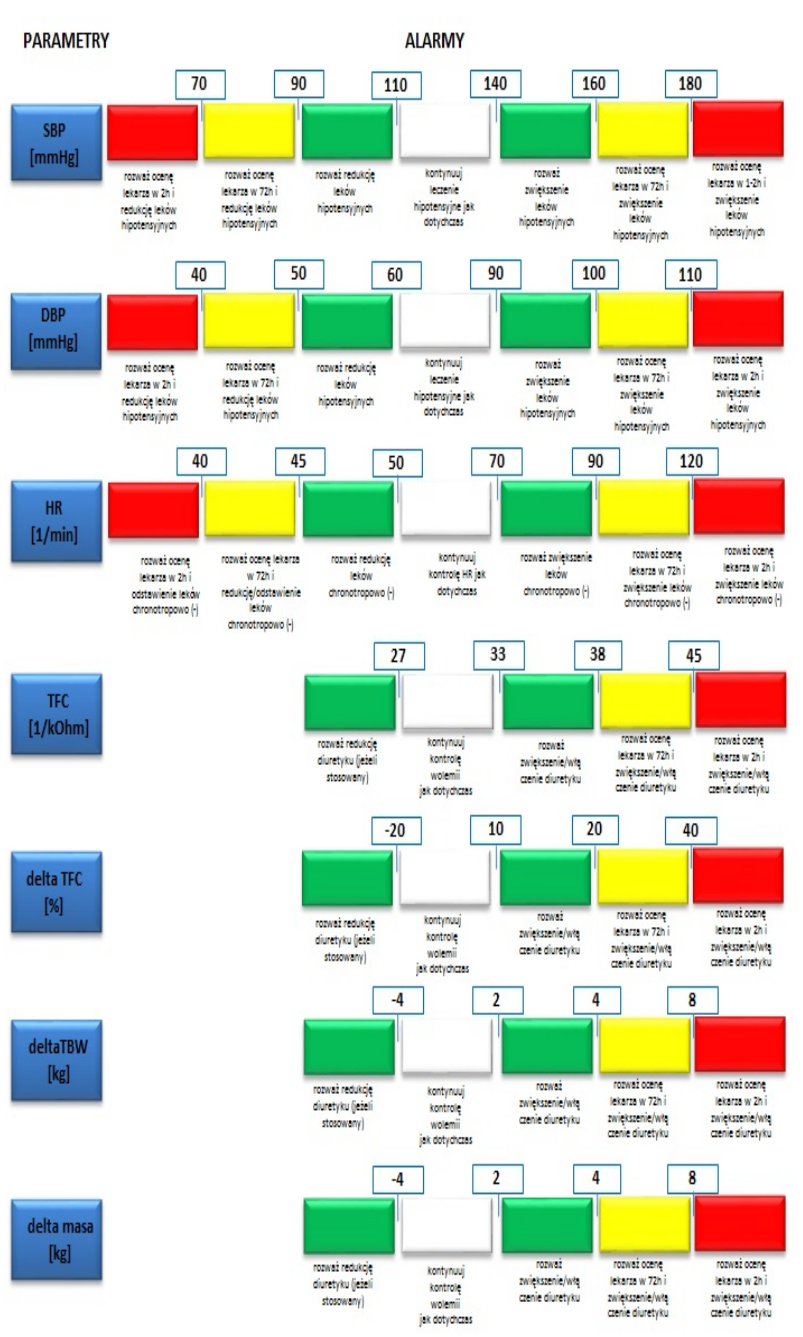

Fig.1. Examples of measured data for cardiology. Each section inside measured value (green, white, yellow, red flag) means in fact separated condition of the observed patient.

\section{Fokker-Planck equation and stochastic resonances}

Let us have a look for master equation with continuous time $t$ and continuous stochastic field $\mathrm{x}$ :

$\frac{\partial P(x, t)}{\partial t}=\int\left(W\left(x \mid x^{\prime}\right) P\left(x^{\prime}, t\right)-W\left(x^{\prime} \mid x\right) P(x, t)\right) d x^{\prime}$

Item $\mathrm{W}\left(\mathrm{x} \mid \mathrm{x}^{\prime}\right)$ and $\mathrm{P}(\mathrm{x}, \mathrm{t})$ means conditional density of probability and density of probability appropriately. When $\mathrm{W}\left(\mathrm{x} \mid \mathrm{x}^{\prime}\right)$ varies itself slowly then one can rewrite it in the form:

$$
W\left(x \mid x^{\prime}\right)=W\left(x^{\prime} \mid r\right) ; \quad r=x-x^{\prime}
$$

Proper form of master equation is as follows:

$$
\begin{aligned}
\frac{\partial P(x, t)}{\partial t}=\int W(x-r ; t) P(x-r, t) d r \\
-P(x, t) \int W\left(x^{\prime} ;-r\right) d x^{\prime} d r
\end{aligned}
$$

When $r$ is small the Tailor series allow us to write:

$$
\begin{aligned}
\frac{\partial P(x, t)}{\partial t}=-\int r & \frac{\partial}{\partial x}(W(x, r) P(x, t)) d r \\
& +\frac{1}{2} \int r^{2} \frac{\partial^{2}}{\partial x^{2}}(W(x, r) P(x, t)) d r
\end{aligned}
$$

Short form is known as Fokker-Planck equation:

$$
\frac{\partial \mathrm{P}(\mathrm{x}, \mathrm{t})}{\partial \mathrm{t}}=-\frac{\partial}{\partial \mathrm{x}}[\mathrm{A}(\mathrm{x}) \mathrm{P}(\mathrm{x}, \mathrm{t})]+\frac{1}{2} \frac{\partial^{2}}{\partial \mathrm{x}^{2}}[\mathrm{~B}(\mathrm{x}) \mathrm{P}(\mathrm{x}, \mathrm{t})]
$$

As $\mathrm{A}$, and $\mathrm{B}$ we describe so called first and second jump moments per time unit as in Feller work [3].

$$
\begin{aligned}
& A(x)=\int r W(x, r) d r \\
& B(x)=\int r^{2} W(x, r) d r
\end{aligned}
$$

Such Form of FP equation can be solved for many situations. Most known is white noise case when stochastic variable varies with autocorrelation

$$
\langle\Delta \mathrm{x}(\mathrm{t})\rangle=0,
$$

and correlation

$$
\left\langle\Delta x(t) \Delta x\left(t^{\prime}\right)\right\rangle=\sigma^{2} \delta\left(t-t^{\prime}\right)
$$

Then FP equation transforms into:

$$
\frac{\partial \mathrm{P}(\mathrm{x}, \mathrm{t})}{\partial \mathrm{t}}=\mathrm{D} \frac{\partial^{2} \mathrm{P}(\mathrm{x}, \mathrm{t})}{\partial \mathrm{x}^{2}}
$$

Diffusion constant results from stochastic process dispersion $\mathrm{D}=\frac{\sigma^{2}}{2}$.

It is easy to verify that for such process $(\mathrm{dx})^{2} / \mathrm{dt}=$ const=D and creates process of the form called normal diffusion:

$$
d x(t)=(2 D)^{1 / 2} d W(t)
$$

$(\mathrm{W}(\mathrm{t})$ is Wiener process).

Different rules for $\mathrm{A}(\mathrm{x})$ and $\mathrm{B}(\mathrm{x})$ appears while kind of noise varies in time, and different solution as well. Solution like drifted diffusion or Orstein-Uhlenbeck process while system oscillates around averaged position is quite well known. Stochastic equation describing such situation is usually Ito equation or 
adequately Langevin equation in physics. Basic conclusion is that the noise shape decides of FP equation form and solution.

\section{Subdiffusion}

It is convenient to use potential well $\varepsilon(\mathrm{x})$ model as is shown in the figure 2 for clarity of explanations. Patient condition can be described by means of set of parameters divided into sections (see fig.1). Because of that each potential well suits such single section.

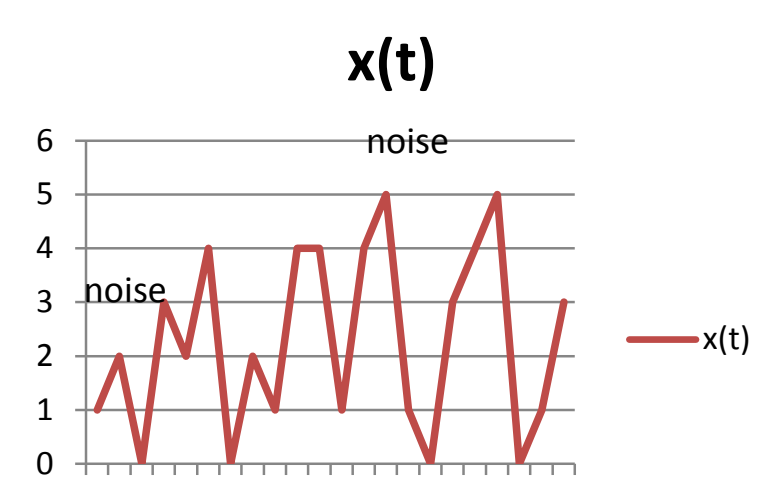

$\begin{array}{llllllllll}1 & 3 & 5 & 7 & 9 & 11 & 13 & 15 & 1719 & 21\end{array}$

Fig.2. Schema of hypothetic possible potential well $\varepsilon(\mathrm{x})$. Lines can point out separate levels of noise.

Shapes of potential wells are stochastic. We are looking for two values:

1. Probability $P_{\varepsilon}(x, t)$ that patient condition jumps from one well to another.

2. Distribution of averaged duration time for unchanged patient condition inside particular well $\Phi(\varepsilon \mid t)$.

Noise causes unexpected phenomena very often. One of most strange is particles diffusion in potential well during absolutely absence of field (means also force) strong enough to do it. Such phenomena are caused by noise presence and are known as stochastic resonances $[3,4]$. In the figure 2 different levels of noise has been sketched to illustrate, that noise in medicine depends on each patient separately, and is patient personal feature. Noise can stabilize system state as well $[5,6]$. What in fact could be noise in patient treatment? Enumerative list could be like that:

1. Medicament driving interaction in patient illness,

2. Influence of genetic properties of patient,

3. Environment impact on patient condition,
4. Patient immunity.

Mentioned factors are not possible of course, and can act in the same time, separately, in accordance, in opposition, and so on. Math model for all possible combinations could be imaginable but it is out of sense to create such models.

Noise influence in form of stochastic resonance causes so called anomaly diffusion of stochastic states. More common name of this phenomenon is subdiffusion or superdiffusion. It was observed in physical experiments [7]. It is also presented in theory of complex net [8]. Subdiffusion differs from normal diffusion joined with Wiener process in formula (11). Adequate stochastic equation for subdiffusion is of the form:

$$
\left\langle d x^{2}(t)\right\rangle=D_{\alpha} t^{\alpha}
$$

Normal diffusion produces Gaussian probability distribution while subdiffusion contains wider class of Levy' processes, and possible result is nongaussian distribution of probability.

Let us assume that:

$$
\rho(\bar{\varepsilon})=\operatorname{Aexp}\left(-\frac{\varepsilon}{\bar{\varepsilon}}\right)
$$

describes distribution of wells in model of potential wells (figure 2). In this formula we used:

$$
\bar{\varepsilon}=\int_{0}^{\infty} \varepsilon \rho(\varepsilon) d \varepsilon
$$

Time of $\varepsilon$ state occupation is distributed in accordance with the form:

$$
\varphi(\mathrm{t})=\int_{0}^{\infty} \Phi(\mathrm{t} \mid \varepsilon) \rho(\varepsilon) \mathrm{d} \varepsilon
$$

In queue theory $\Phi(t \mid \varepsilon)$ is interchange time or time between transactions. Here we will call it "unconditional waiting time". Obvious rule is fulfilled:

$$
\int_{0}^{\infty} \varphi(\mathrm{t}) \mathrm{dt}=1
$$

Average time value for patient "waiting inside potential well" is:

$$
\langle t\rangle=\int_{0}^{\infty} t \varphi(t) d t
$$

Let us take into account two particular situations:

1. Uniform wells distribution $\rho(\bar{\varepsilon})=\frac{1}{2 \Delta}$ while unconditional waiting time is as follows $\Phi(t \mid \varepsilon)=\frac{1}{\tau(\varepsilon)} \exp \left(\frac{t}{\tau(\varepsilon)}\right) \quad, \quad$ and $\quad \tau(\varepsilon)=$ $\tau_{0} \exp (\beta \varepsilon)$, where $\beta=1 / k T$ 
2. Laplace' well distribution $\rho(\varepsilon)=$ $\frac{1}{2 \sigma} \exp (-|\varepsilon| / \sigma)$ where $\sigma=\langle|\varepsilon|\rangle$

Results are placed appropriately below:

1. $\varphi(t)=\frac{1}{2 \Delta} \frac{1}{\beta t}\left[\exp \left(\frac{-t}{\tau_{+}}\right)-\exp \left(\frac{-t}{\tau_{-}}\right)\right]$while $\tau_{ \pm}=\tau_{0} \exp (-\beta \Delta)$, and

$\langle t\rangle=\tau_{c}=\tau_{0} \frac{\cosh (\beta \Delta)}{\beta \Delta}$

2. $\varphi(t)=\frac{1}{2 \sigma \beta \tau_{0}}\left[\left(\frac{\tau_{0}}{t}\right)^{1+\frac{1}{\sigma \beta}} \gamma\left(1+\frac{1}{\sigma \beta}, \frac{t}{\tau_{0}}\right)+\right.$ $\left.\left(\frac{\tau_{0}}{t}\right)^{1-\frac{1}{\sigma \beta}} \Gamma\left(1-\frac{1}{\sigma \beta}, \frac{t}{\tau_{0}}\right)\right], \quad$ and $\langle t\rangle=\tau_{c}=\frac{\tau_{0}}{1-(\beta \sigma)^{2}}$

Item $\gamma$ and $\Gamma$ in (19) are Euler functions. Both results are different, and in second case one can observe kind of phase transition in dynamics of system. So different potential wells distribution produces quite unlike system behavior. In both cases we observe characteristic feature that $\varphi(t)$ vanishes like "fat tail" distribution. So generally $\sim \mathrm{t}^{-\alpha}$ can be expected as a rule in subdiffusion processes.

We used

$$
\frac{\mathrm{kT}}{\bar{\varepsilon}}=\beta \bar{\varepsilon}
$$

Expression kT describes thermal energy of thermostat what means energy from around. For general stochastic system one can assume it as pool averaged value of the measured parameter. Similarly average energy $\varepsilon$ can be treated in our observations as spread of the measured parameters. Of course such assumptions must properly fit all observed values in patient treatment. So $\beta$, and $\varepsilon$ are created appropriately for medical observations. One must have in mind that product $\beta \varepsilon$ is always dimension less.

\section{Nonadditive entropies approach and general view of subdiffusion}

Because of very particular examples (formulae 18 and 19) more general solution should be presented. For complex systems with possible internal interaction entropy $\mathrm{S}$ can be described in form:

$$
S^{i}(A+B)=S^{i}(A)+S^{i}(B)+(1-q) S^{i}(A) S^{i}(B)
$$

Item $\mathrm{A}$, and $\mathrm{B}$ are two different component of complex system with unknown interaction between them. Item $\mathrm{q}$ $(q \neq 1)$ is called fractional coefficient. Index $i$ is for exact model of nonadditive entropy.

Stream of probability is defined as:

$$
J=Q f(P) \frac{\partial}{\partial x} \frac{\delta S}{\delta P}
$$

Coefficient Q contains all possible fluctuations while $\mathrm{f}(\mathrm{P})$ describes those arising from fluctuations of $\mathrm{P}$, and $\mathrm{S}$ is entropy. Stream must fulfill continuity rule:

$$
\frac{\partial J(x, t)}{\partial x}=-\frac{\partial P(x, t)}{\partial t}
$$

Resulting nonlinear and fractional Fokker-Planck equation is as follows [9-15]:

$$
\frac{\partial P(x, t)}{\partial t}=\frac{\partial^{2} P^{q}(x, t)}{\partial x^{2}}
$$

Exponent q depends on exact form of entropy S. For Tssallis entropy [16-18]

$$
S_{q}(P)=\frac{1-\int_{0}^{\Omega} P^{q}(x) d x}{q-1}
$$

we obtain FP equation of the form:

$$
\frac{\partial P(x, t)}{\partial t}=Q_{T} \frac{\partial^{2} P^{q}(x, t)}{\partial x^{2}}
$$

So called Barrenblatt solution [19] of eq. 26 with initial condition $P(x, 0)=\delta(t)$ takes the form:

$$
P^{q}(x, t)=\frac{1}{t^{k}}\left(\left\{1-\frac{k(q-1)}{2 q N} \frac{|x|^{2}}{t^{\frac{2 k}{N}}}\right\}_{+}\right)^{1 / q-1}
$$

In formula 27 item $\mathrm{N}$ is math space dimension, and $k=\left(q-1+\frac{2}{N}\right)^{-1}$. We can see that "fat tail" distribution is property of general result as well as in mentioned above examples for time distribution calculations.

\section{Resume}

Proposed approach for patient condition math model exploits theory of stochastic resonances, subdiffusion which is common property of such resonances, and more general nonadditive entropy theory in complex systems. All are known from physics of complex systems, and dynamics of such systems. We use some thermodynamics values in math forms but we propose how one can generalize them into stochastic processes 
which are rather far from thermodynamics (comments after formula 20) . Earlier similar approach in complex system proposed Vazquez and his coauthors [20] also far from thermodynamics. It is well known now that many properties observed preliminary only in physics of condensed matter are common features of complex systems in finance, internet, and so on.

Patient health is obviously complex system too. Most interesting result seems to us that probabilities in patient condition prediction behave as "fat tail" rather so near to Levy processes. We illustrate by examples that "expected time in potential well" or in other words expected time without any measured change of patient condition strongly depends on generally unknown potential wells distribution.

We also step by step explained how Fokker - Planck equation arises with its noise dependent solutions. This was added for the sake of all lecture clarity. Noise shape strongly influences on FP solutions, and in fact explains how and why nonlinear fractional form of FP equation arises as well.

Merits of proposed approach are continuous model which allow us to get form of probability of jump between states of patient health. It can be obtained even if medical description of patient states is in fact linear. Proposed solution is general, independent on measured patient parameter. Results will be applied in IT system we are built for ICG measurements for patient care in mobile, telemedicine platform.

\section{References}

1. Palmer S, Sculpher M, Phillips Z, Robinson M, Ginnelly L, Bakhai A et al. Management of non-ST elevation acute coronary syndrome: how cost-effective are glycoprotein IIb/IIIa antagonists in the U.K. National Health Service?. International $J$ Cardiology $\mathbf{1 0 0}$ (2005) 229-40

2. https://amulet.wim.mil.pl

3. Feller, W. (2000). An Introduction to Probability Theory and its Applications, John Wiley and Sons, New York and Chichester, vol.2

4. P. Hänggi, P. Talkner, and M. Borkovec. Reaction-rate theory: Fifty years after Kramers, Rev. Mod. Phys., 62:251-341, (1990)

5. H. A. Kramers. Brownian motion in a field of force and the diffusion model of chemical reaction, Physica (Utrecht), 7:284-304, (1940)
6. N. V. Agudov and B. Spagnolo, Noiseenhanced stability of periodically driven metastable states, Phys. Rev. E, 64:035102-14(R), (2001)

7. W. Horsthemke and R. Lefever. Noiseinducted transitions. Theory and applications in physics, chemistry, and biology, Springer Verlag, Berlin, (1984)

8. A.Fronczak, P. Fronczak Biased random walks on complex networks: the role of local navigation rules arxiv:0709.2231 (Sept. 2007)

9. T.D. Frank, A Langevin approach for the microscopic dynamics of nonlinear FokkerPlanck equations, Physica A 301, 52-62, (2001). doi:10.1016/S0378-4371(01)00345-4

10. T.D. Frank, Generalized Fokker-Planck equations derived from generalized linear nonequilibrium thermodynamics, Physica A 310, 397-412, (2002). doi:10.1016/S03784371(02)00821-X

11. T.D. Frank, Stochastic feedback, nonlinear families of Markov processes, and nonlinear Fokker-Planck equations, Physica A 331, 391-408, (2004). doi:10.1016/j.physa.2003.09.056

12. T.D. Frank, Nonlinear Fokker-Planck Equations. Fundamental and Applications, Springer (2005)

13. T.D. Frank, Nonextensive cutoff distributions of postural sway for the old and the young, Physica A 388, 2503- 2510, (2009). doi:10.1016/j.physa.2009.03.003

14. T.D. Frank, A. Daffertshofer, Exact timedependent solutions of the Rényi FokkerPlanck equation and the Fokker-Planck equations related to the entropies proposed by Sharma and Mittal, Physica A, 285, 351-366, (2000). doi:10.1016/S0378-4371(00)00178-3

15. T.D. Frank, R. Friedrich, Estimating the nonextensivity of systems from experimental data: a nonlinear diffusion equation approach, Physica A 347, 65-76, (2005). doi:10.1016/j.physa.2004.08.031

16. C. Tsallis, Nonextensive statistics: theoretical. Experimental and computational evidences and connections, Braz. J. Phys. 29, 1-35, (1999). doi:10.1590/S010397331999000100002

17. C. Tsallis, Introduction to Nonextensive Statistical Mechanics, Springer (2009)

18. C. Tsallis, D. Bukman, Anomalous diffusion in the presence of external forces: exact timedependent solutions and their rhermostatical 
basis Phys. Rev. E 54, R2197-R2200, (1996). doi:10.1103/PhysRevE.54.R2197

19. Z. Wu, J. Zhao, J. Yim, H. Li, Nonlinear diffusion equations, World Scientific, (2001)
20. A. Vazquez, J. G. Oliveira, Z. Dezso, K.-I. Goh, I. Kondor, and A.-L. Barabasi, $\begin{array}{lllll}\text { Phys.Rev. } & \text { E } & \text { 73, } & 036127 & \text { (2006) }\end{array}$ 